OPEN ACCESS

Edited by:

Paul T. P. Wong,

Trent University, Canada

Reviewed by:

Karen Henry,

Henry, United States

Theodore C. K. Cheung, University of Toronto Scarborough,

Canada

*Correspondence:

David E. Reed II

david.reed7@va.gov

Specialty section:

This article was submitted to

Health Psychology,

a section of the journal

Frontiers in Psychology

Received: 19 December 2020

Accepted: 06 April 2021

Published: 28 April 2021

Citation:

Reed DE II, Lehinger E, Cobos B,

Vail KE III, Nabity PS, Helm PJ, Galgali MS and McGeary DD (2021)

Authenticity as a Resilience Factor Against CV-19 Threat Among Those With Chronic Pain and Posttraumatic

Stress Disorder.

Front. Psychol. 12:643869. doi: 10.3389/fpsyg.2021.643869

\section{Authenticity as a Resilience Factor Against CV-19 Threat Among Those With Chronic Pain and Posttraumatic Stress Disorder}

\author{
David E. Reed II,2*, Elizabeth Lehinger ${ }^{3}$, Briana Cobos ${ }^{2,4}$, Kenneth E. Vail III5, \\ Paul S. Nabity' ${ }^{2}$ Peter J. Helm ${ }^{6}$, Madhwa S. Galgali ${ }^{6}$ and Donald D. McGeary2,7 \\ ${ }^{1}$ Center of Innovation for Veteran-Centered and Value-Driven Care, VA Puget Sound Health Care, Seattle, WA, \\ United States, ${ }^{2}$ Department of Psychiatry and Behavioral Sciences, Texas Health Science Center at San Antonio, San \\ Antonio, TX, United States, ${ }^{3}$ Department of Psychiatry and Behavioral Sciences, University of Washington, Seattle, WA, \\ United States, ${ }^{4}$ Department of Psychology, University of Texas at San Antonio, San Antonio, TX, United States, \\ ${ }^{5}$ Department of Psychology, Cleveland State University, Cleveland, OH, United States, ${ }^{6}$ Department of Psychological \\ Sciences, University of Missouri, Columbia, MO, United States, ${ }^{7}$ Department of Rehabilitation Medicine, University of Texas \\ Health Science Center at San Antonio, San Antonio, TX, United States
}

Objective: The novel coronavirus (2019; CV-19) is linked to increases in emotional distress and may be particularly problematic for those with pre-existing mental and physical conditions, such as chronic pain and posttraumatic stress disorder (PTSD). However, little empirical research has been published on resilience factors in these individuals. The present study aims to examine authenticity as a resilience factor among those with chronic pain and/or PTSD.

Methods: Prior to the national response to the pandemic (January 10-24, 2020), participants were screened for pain-related disability (Oswestry Disability Index; ODI) and PTSD symptoms (Posttraumatic Checklist for DSM-5; PCL-5), and on the basis of those responses were categorized into one of four groups: healthy, chronic pain only, PTSD only, or comorbid chronic pain and PTSD. During the CV-19 pandemic (May 5May 13, 2020), participants responded again to the ODI and PCL-5, in addition to the Wood Authenticity Scale, Brief Pain Inventory, and items related to the CV-19 pandemic.

Results: A total of 110 participants (54.55\% women), aged 42.19 ( $S D=13.16$ ), completed the survey during the pandemic. The comorbid group endorsed higher levels of CV-19 Threat and Impact compared to all other groups. Authenticity moderated this relationship relevant to CV-19 Threat among those in the chronic pain only group, and not in any other group.

Conclusion: The comorbid group endorsed higher levels of CV-19 Threat and Impact compared to all other groups. Importantly, greater authenticity was associated with less CV-19 Threat in the chronic pain only group, and not in any other group. The present study also highlights the importance of engaging authentically for those with chronic pain during the pandemic.

Keywords: COVID-19 pandemic, CV-19 threat, CV-19 impact, authenticity, pain-related disability, PTSD 


\section{INTRODUCTION}

The novel coronavirus (2019; CV-19) has claimed the lives of over 550,000 individuals in the United States (World Health Organization [WHO], 2020). In addition, increases in suicidality, anxiety, and depression have been documented during the pandemic (Czeisler et al., 2020), highlighting the pandemic's psychological toll. Individuals with physical or psychological pre-existing conditions, such as chronic pain (defined as experiencing pain for at least 3 months) and/or posttraumatic stress disorder (PTSD), may be significantly impacted by the pandemic (Merskey and Bogduk, 1994; Treede et al., 2015; Pfefferbaum and North, 2020). Nevertheless, little empirical research has been published specifically on these populations during the pandemic. Moreover, there is a paucity of research on understanding how resilience factors may help patients with chronic pain and PTSD navigate through the effects of the pandemic. Resilience is adapting to novel stressors in a way that prevents long-term mental health issues (Kalisch et al., 2017). It is a learned set of behaviors, not an innate factor that cannot be changed (Kalisch et al., 2017). Capturing data on putative resilience factors in the midst of adversity, such as the current CV-19 pandemic, is critical to our understanding of realworld resilience in these populations. The present work aims to fill this gap by assessing authenticity as a resilience factor among those with chronic pain only, PTSD only, and comorbid chronic pain and PTSD.

Among the general population, research has focused on CV-19 pandemic-specific anxieties, in addition to $\mathrm{CV}-19$ pandemicspecific financial and emotional burdens (Conway et al., 2020; Guo et al., 2020; Liu et al., 2020), defined by Conway et al. (2020) as CV-19 Threat and CV-19 Impact, respectively. The financial impact of the CV-19 pandemic among the general population is linked to higher symptoms of PTSD and depression (Guo et al., 2020), and anxiety specific to CV-19 has been linked to depression, anxiety, alcohol use, and PTSD (Liu et al., 2020; Rodriguez et al., 2020).

Individuals with mental and physical health conditions are constantly managing the stress of their disorders. The physiological and psychological burden, or "allostatic load," may result in difficulties processing and managing additional stressors (McEwen, 1998; Sterling and Eyer, 1988). For example, pain-related disability worsens when depression or PTSD is also included in the clinical picture along with pain (Benedict et al., 2020; McGeary et al., 2020), and cardiovascular and metabolic issues are linked to PTSD (Ryder et al., 2018). The pandemic serves as an additional stressor for those with chronic pain and/or PTSD. Therefore, individuals with more severe mental and physical health symptoms may endorse more severe perceptions of CV-19 anxiety and CV-19 impact. However, because of authenticity's positive effects on mood (Grijak, 2017), feeling authentic may promote resilience and help ameliorate these effects.

Resilience is an adaptive and dynamic process, whereby stressors result in cognitive, affective, and behavior changes that promote mental health (Kalisch et al., 2017, 2019). In a way, resilience is about either developing and enacting one's new authentic self, or ensuring that one's authentic self is not affected negatively. Authenticity is defined as congruence between one's internal physiological states (e.g., feelings of happiness), accurate awareness of these states, and engaging in behaviors that are appropriate to those internal states and corresponding awareness (Wood et al., 2008). Inauthenticity may be felt when internal psychological states do not match with one's awareness of these states (i.e., self-alienation) and/or when awareness of these states are not congruent with behaviors (i.e., inauthentic living; Wood et al., 2008). As such, authenticity entails emotional states, cognitions, and behaviors. Higher levels of authenticity have been linked to increased psychological well-being, decreased anxiety, and decreased depression (Grijak, 2017).

In a longitudinal study examining authenticity, life satisfaction, and distress across two time points, Boyraz et al. (2014) found that authenticity predicted greater life satisfaction and less distress six weeks later. Moreover, the relationships between authenticity, life satisfaction, and distress were all significant at Time 2 , suggesting that authenticity served as a concurrent and future resilience factor. Results are congruent with cross-sectional studies showing that authenticity is linked to lower levels of anxiety, depression, and loss of control, and greater meaning in life and psychological well-being (Vess et al., 2016; Grijak, 2017).

Theorists posit that one's mortality is at the core of the distress related to the CV-19 pandemic (Pyszczynski et al., 2020). Although viewing one's future as limited may promote negative affect (Grühn et al., 2016), it is also an opportunity to harness positive outcomes (Vail et al., 2012; Reed, 2020). Authenticity may buffer against negative affect associated with limited time perspectives in the future during the pandemic. Indeed, Davis and Hicks (2013) published a series of experimental studies showing how authenticity helps promote hope when experiencing limited time perspectives. In addition, Bryan et al. (2017) found that authenticity attenuated the relationship between loneliness and depression, anxiety, and physical symptoms. A recent longitudinal study provided compelling evidence for authenticity as a buffer against collective trauma within the context of Hurricane Harvey. The events of Hurricane Harvey, similar to the CV-19 pandemic, affected a large group of individuals and significantly altered their way of living. Among a sample of individuals of which many had experienced Hurricane Harvey, Maffly-Kipp et al. (2020) showed that inauthenticity at an earlier time point contributed to higher stress at a later time point. These results are congruent with the longitudinal analyses of Boyraz et al. (2014) and showed that stressors that alter daily existence are attenuated by perceived authenticity. Authenticity as a resilience factor may be particularly crucial for individuals who are forced to alter their preferred way of being due to mental or physical health conditions, such as those with chronic pain and/or PTSD.

Individuals diagnosed with chronic pain and/or PTSD potentially experience a disruption of their sense of self, often precipitated by the loss of social roles (e.g., employment) and an inability to engage in life as they desire (Morley, 2008; Mitchell et al., 2020; Packham et al., 2020). Avoidance, social isolation, and/or pain-related disability may prevent individuals from engaging in behaviors that provide a sense of authenticity 
(i.e., engaging in life consistent with how one views themselves; Wood et al., 2008). This is particularly problematic because lower levels of authenticity are associated with more distress, anxiety, and depression, and lower levels of well-being (Sheldon et al., 1997; Grijak, 2017).

Chronic pain does not merely interfere with one's life; individuals with chronic pain experience a disruption of identity and self-definition (Morley, 2008). Indeed, chronic pain may be considered an external influence that potentially disrupts one's perceived authenticity, as described by Wood et al. (2008). Adults with chronic pain have difficulty recognizing their emotions, which is associated with higher pain severity, pain disability, and distress (Aaron et al., 2019; Blaettler et al., 2019). In turn, this may result in individuals with chronic pain acting in ways that are not congruent with how they view themselves (e.g., not engaging with their grandchildren due to pain; Smith and Osborn, 2007), thus contributing to inauthenticity. Moreover, individuals may experience their emotions and thoughts associated with chronic pain as inauthentic. In all, chronic pain acts as a problematic external influence that forces individuals to engage in life in a different way relative to their premorbid and/or preferred emotional, physical, and social functioning (McWilliams et al., 2003; Morley, 2008; De Heer et al., 2014; Packham et al., 2020).

Similarly, PTSD is defined by avoidance of internal and external trauma reminders, negative alterations in cognitions and mood, hypervigilance, and intrusive symptoms (American Psychiatric Association [APA], 2013). Avoiding previously valued behaviors (e.g., going to play in a park or going out to eat) in the service of preventing thoughts and emotions related to a traumatic experience may be felt as an inauthentic behavior. In addition, loss of trust and security, both part of the PTSD sequelae (Bell et al., 2019; Ehlers and Clark, 2000), may prevent individuals from engaging with others in a way that feels natural and authentic.

Individuals with comorbid chronic pain and PTSD are particularly susceptible to disruptions of identity (Reed et al., 2021). Not only are they managing the symptoms of a physical and mental health disorder simultaneously, but symptoms of pain and PTSD may maintain each other (mutual maintenance model; Sharp and Harvey, 2001). For instance, Feinberg et al. (2017) found that hyperarousal and intrusion symptoms at 6 weeks after an emergency department (ED) visit mediated the relationship between pain symptoms during the ED visit and pain symptoms 1 year after the ED visit. Moreover, individuals with chronic pain and PTSD endorse higher levels of pain severity, depression, and healthcare utilization compared to those with pain but without PTSD (Benedict et al., 2020). In line with the theory of allostatic load, it is likely that individuals with comorbid chronic pain and PTSD would experience the pandemic differently than those with one or none of these disorders, endorsing higher levels of CV-19 Threat and Impact. Living authentically may ameliorate CV-19 Threat and Impact among this population, however.

If engaging in life authentically is associated with positive outcomes (Grijak, 2017), then individuals with higher levels of authenticity would presumably be protected from anxieties and burdens associated with the pandemic. Therefore, we hypothesized that authenticity would be associated with lower levels of CV-19 Threat and Impact. The addition of chronic pain, PTSD, or comorbid chronic pain and PTSD increases one's allostatic load and vulnerability to an additional stressor, such as the pandemic. Therefore, we hypothesized that the relationship between authenticity and CV-19 Threat and Impact would be weaker (i.e., an interaction between group and authenticity) for individuals in the chronic pain only and PTSD only groups (compared to the healthy group) and in the comorbid chronic pain and PTSD only group (compared to all other groups).

\section{METHOD}

\section{Procedure}

The present research utilized data collected during the CV-19 pandemic as part of a larger longitudinal quasi-experimental study. Data were collected using CloudResearch (Litman et al., 2017), a web interface designed to more easily recruit reliable and valid online data from several clinical populations. For overall study design ${ }^{1}$. Prior to the national response to the pandemic (January 10-24, 2020), participants were screened for pain-related disability (Oswestry Disability Index; ODI) and PTSD symptoms (Posttraumatic Checklist for DSM-5; PCL-5), and on the basis of those responses were categorized into one of four groups. The healthy group was comprised of individuals in the lower quartile on the ODI (8\% pain disability or less) and PCL-5 (15 or less). The chronic pain only group endorsed experiencing pain for at least 3 months and at least $21 \%$ pain disability (which is indicative of moderate disability associated with everyday movements; Michigan State University, 2020) and in the lower quartile on the PCL-5. Individuals in the PTSD only group were in the lower quartile of pain disability and endorsed 37 or higher on the PCL-5. Research suggests 37 is an optimal cut-off score when determining a PTSD diagnosis when over-diagnosing is a concern (i.e., high specificity; Blevins et al., 2015). Individuals in the comorbid chronic pain only and PTSD only group endorsed moderate to severe pain disability and at least 37 on the PCL-5. If individuals did not fall into these categories, they were excluded from participation. For consort chart ${ }^{2}$. For the present study, only scores collected at Time 2 (during the CV-19 pandemic) were used. For more detailed information on the specifics of the present study design, including pre-registered hypotheses ${ }^{3}$. The present study was completed with approval from the University of Texas Health Science Center IRB.

\section{Participants}

A total of 110 participants (60 women; 54.55\%) completed assessments during the CV-19 pandemic between May 5 and May 13, 2020. On average, participants were $42.19(S D=13.16)$ years old, with an age range of 22 to 82 . There were statistically significant differences in age across groups, $F(3,106)=7.73$, $p<0.001$, with Tukey's HSD bias correction indicating the chronic pain group $(M=50.18 ; S D=13.39)$ was older than the

\footnotetext{
${ }^{1}$ https://osf.io/hsyxv

${ }^{2}$ https://osf.io/a35m4/

${ }^{3}$ https://osf.io/9zbe2
} 
healthy group $(M=37.66 ; S D=12.99$; difference $=12.52[95 \% C I$ : $4.75,20.29], p<0.001)$, PTSD group $(M=37.18 ; S D=10.61$; difference $=-12.99$ [95\% CI: $-21.63,-4.36], p=0.001)$, and comorbid group $(M=41.45 ; S D=9.70$; difference $=-8.72[95 \%$ $C I:-17.36,-0.09], p=0.047)$. Participants were predominantly White $(n=94 ; 85.45 \%)$ and either had a 4 -year college degree $(n=34 ; 30.91 \%)$ or some college $(n=29 ; 26.36 \%)$ or. See Table 1 for all sample characteristics.

\section{Measures}

\section{Demographics}

Participants provided information related to gender, age, race, and education.

\section{CV-19 Threat}

Participants completed a 6-item questionnaire measuring how threatened or worried they were about CV-19 on a 7-point scale ( $1=$ "Not True of Me At All" to $7=$ "Very True of Me"). Examples of questions include "Thinking about the coronavirus (COVID-19) makes me feel threatened" and "I am afraid of the coronavirus" (Conway et al., 2020). Internal consistency of CV-19 Threat for the present study was $\alpha=0.90$.

\section{CV-19 Impact}

Participants completed a 9-item assessment measuring how participants were financially and psychologically impacted by the coronavirus on a 7-point scale $(1=$ "Not True of Me At All" to $7=$ "Very True of Me"). Questions included: "The Coronavirus (COVID-19) has impacted me negatively from a financial point of view" and "I have lost job-related income due to the Coronavirus." Items were summed for data analysis (Conway et al., 2020). Internal consistency of CV-19 Impact for the present study was $\alpha=0.85$.

\section{Authenticity}

The Wood Authenticity Scale is a 12-item assessment that measures three components of authenticity: authentic living, accepting external influence, and self-alienation (Wood et al., 2008). An example item is, "I am true to myself in most situations," and participants responded on a 7-point scale $(1=$ "Does not describe me at all" to $7=$ "Describes me very well"). Items were summed for a total score, and the internal consistency for the present study was $\alpha=0.91$.

\section{Pain Disability}

A modified version of the Oswestry Disability Index (ODI) measured an individual's perception of disability due to pain

TABLE 1 | Sample characteristics.

\begin{tabular}{|c|c|c|c|c|c|c|}
\hline & Healthy & Chronic Pain & PTSD & Comorbid & $p$ & SMD \\
\hline & $\begin{array}{c}\text { Mean or Freq. SD or } \\
\text { Percentage }\end{array}$ & $\begin{array}{c}\text { Mean or Freq. SD or } \\
\text { Percentage }\end{array}$ & $\begin{array}{c}\text { Mean or Freq. SD or } \\
\text { Percentage }\end{array}$ & $\begin{array}{c}\text { Mean or Freq. SD or } \\
\text { Percentage }\end{array}$ & & \\
\hline$n$ & 32 & 34 & 22 & 22 & & \\
\hline Age & 37.66 (12.99) & 50.18 (13.39) & $37.18(10.61)$ & $41.45(9.70)$ & $<0.001$ & 0.594 \\
\hline Race & & & & & 0.048 & 0.646 \\
\hline African American & $3(9.4)$ & $0(0.0)$ & $1(4.5)$ & $2(9.1)$ & & \\
\hline Asian & $6(18.8)$ & $0(0.0)$ & $0(0.0)$ & $2(9.1)$ & & \\
\hline Decline to Answer & $0(0.0)$ & $0(0.0)$ & $0(0.0)$ & $1(4.5)$ & & \\
\hline Hispanic & $0(0.0)$ & $1(2.9)$ & $0(0.0)$ & $0(0.0)$ & & \\
\hline White/Caucasian & $23(71.9)$ & $33(97.1)$ & $21(95.5)$ & $17(77.3)$ & & \\
\hline Gender & & & & & 0.570 & 0.220 \\
\hline Decline to Answer & $0(0.0)$ & $0(0.0)$ & $0(0.0)$ & $1(4.5)$ & & \\
\hline Female & $18(56.2)$ & $17(50.0)$ & $12(54.5)$ & $13(59.1)$ & & \\
\hline Male & $14(43.8)$ & $17(50.0)$ & $10(45.5)$ & $8(36.4)$ & & \\
\hline Sexual Orientation & & & & & 0.148 & 0.449 \\
\hline Bisexual & $2(6.2)$ & $2(5.9)$ & $5(22.7)$ & $2(9.1)$ & & \\
\hline Gay or Lesbian & $1(3.1)$ & $0(0.0)$ & $2(9.1)$ & $2(9.1)$ & & \\
\hline Heterosexual & $29(90.6)$ & $32(94.1)$ & $15(68.2)$ & $18(81.8)$ & & \\
\hline Education & & & & & 0.056 & 0.917 \\
\hline 2-year College Degree & $2(6.2)$ & $8(23.5)$ & $4(18.2)$ & $6(27.3)$ & & \\
\hline 4-year College Degree & $17(53.1)$ & $8(23.5)$ & $5(22.7)$ & 4 (18.2) & & \\
\hline Decline to Answer & $0(0.0)$ & $0(0.0)$ & $0(0.0)$ & $1(4.5)$ & & \\
\hline Doctoral Degree & $1(3.1)$ & $0(0.0)$ & $0(0.0)$ & $1(4.5)$ & & \\
\hline High School/GED & $2(6.2)$ & $4(11.8)$ & $4(18.2)$ & $0(0.0)$ & & \\
\hline Less than High School & $1(3.1)$ & $0(0.0)$ & $0(0.0)$ & $0(0.0)$ & & \\
\hline Master's Degree & 5 (15.6) & $1(2.9)$ & $3(13.6)$ & 4 (18.2) & & \\
\hline Some College & $4(12.5)$ & $13(38.2)$ & $6(27.3)$ & 6 (27.3) & & \\
\hline
\end{tabular}

SMD = standardized mean difference. 
using ten different daily life domains, such as lifting, walking, traveling, sitting, standing, sleeping, social life, and employment (Fairbank et al., 1980; Fritz and Irrgang, 2001). Participants completed the 10-item questionnaire on a 0-5 scale. A final percentage score was taken for each time point by taking the average of each item and multiplying that average by 100 . Internal consistency of the ODI for the present study was $\alpha=0.93$.

\section{PTSD}

The Posttraumatic Stress Disorder Checklist for DSM-5 (PCL5) was used to measure PTSD symptoms using the current Diagnostic and Statistical Manual's criteria (Weathers et al., 2013). Individuals were asked to indicate a traumatic event (i.e., Criterion A event) in their lifetime and the extent to which they have been bothered by PTSD symptoms in the past month using a 20 -item rating scale from a 0 to 4 scale $(0=$ "Not at all" to $4=$ "Extremely"). Items were summed for data analysis. Internal consistency of the PCL-5 for the present study was $\alpha=0.96$.

\section{Pain Severity}

The Modified Brief Pain Inventory Short Form (mBPI-SF; Cleeland, 1991) measured the level of the participant's pain severity by averaging 4-items of pain at its worst, least, average, and current pain (i.e., now; 0-10 scale). An example item is, "Please rate your pain by marking the number that best describes your pain at its worst in the last 24 hours." Items were averaged for data analysis. Internal consistency for the mBPI-SF for the present study was $\alpha=0.94$.

\section{Data Analytic Strategy}

In order to address study hypotheses, general linear models (GLM) were examined, one for each outcome (CV-19 Threat and CV-19 Impact), wherein Gaussian distributions were specified. Each model included Group and Authenticity as predictors, in addition to a Group by Authenticity interaction. Visual inspection of data plots indicated that a quadratic relationship may emerge between authenticity and the outcome of interest. Therefore, a quadratic authenticity term was entered into each model to account for curvilinear relationships. Authenticity was grand-mean centered in each model, and the centered variable was used to create the interaction and quadratic terms. Age (grand-mean centered) was included in the model as a covariate due to between-group differences. Regression estimates of GLMs were examined for significance. Correlations between variables of interest are also presented.

\section{RESULTS}

\section{Descriptive Statistics}

Participants in the healthy group $(n=32)$ endorsed low levels of pain severity $(M=0.84 ; S D=1.37)$, pain disability $(M=2.56 \%$; $S D=4.38 \%)$, and PTSD symptoms $(M=4.19 ; S D=5.86)$. Individuals in the chronic pain only group $(n=34)$ endorsed pain severity levels $(M=3.65 ; S D=2.01)$ most consistent with mild pain (Serlin et al., 1995; Jones et al., 2007), and pain disability levels $(M=29.24 \%$; $S D=18.08 \%)$ consistent with individuals with chronic pain. The chronic pain only group endorsed PCL5 scores $(M=8.53 ; S D=10.16)$ consistent with individuals without PTSD. Individuals in the PTSD only group endorsed an average pain severity rating of $2.22(S D=2.06)$ and pain disability of $9.36 \%(S D=10.00 \%)$, consistent with individuals without chronic pain. The PTSD only group endorsed PCL-5 scores slightly below average of what would be consistent with a PTSD diagnosis $(M=30.09 ; S D=17.20)$ but is close to a suggested cut-off score of 31-33 (Blevins et al., 2015). Pre-screening prior to the pandemic indicated PCL-5 scores were consistent with a PTSD diagnosis for those who completed both time points $(M=47.77 ; S D=9.60)$. In addition, recommendations leave room for lowering the cut-off scores depending on the purpose of the assessment (U.S. Department of Veterans Affairs, 2020). Finally, participants in the comorbid group endorsed an average pain severity rating of $3.59(S D=2.27)$, pain disability of $33.18 \%$ $(S D=19.29 \%)$, and PCL-5 score of $38.23(S D=17.65)$, all of which are consistent with individuals who have comorbid chronic pain and PTSD. Average authenticity score was 68.78 $(S D=13.27)$ for the healthy group, $71.68(S D=9.90)$ for the chronic pain only group, $66.59(S D=15.55)$ for the PTSD only group, and $58.36(S D=16.59)$ for the comorbid group. Analysis of variance indicated significant group differences in authenticity (using Tukey's HSD correction; $F[3,106]=4.50, p=0.005$ ), with the comorbid condition endorsing less authenticity than the healthy group (difference $=-10.42$ [95\% CI: $-20.24,-0.60]$, $p=0.033$ ) and chronic pain only group (difference $=-13.31[95 \%$ CI: $-23.01,-3.61$ ], $p=0.003)$.

\section{Outcomes}

\section{CV-19 Threat}

A main effect of group emerged with CV-19 Threat as the outcome, $F(3,100)=2.79, p=0.044$, partial $\eta^{2}=0.05$. Regression analyses indicated that individuals in the comorbid group endorsed more CV-19 Threat compared to individuals in the healthy group $(b=6.98, S . E .=2.78, p=0.014,95 \% C I: 1.52,12.43$, $\beta=0.29)$. The chronic pain only group $(b=1.00$, S.E. $=2.61$, $p=0.703$, 95\% CI:-4.12, 6.12, $\beta=0.05)$ and PTSD only group $(b=-0.99$, S.E. $=2.54, p=0.697,95 \%$ CI:-5.96, 3.98, $\beta=-0.04)$ did not differ in CV-19 Threat compared to the healthy group. Estimated marginal means are shown in Table 2. When Tukey's HSD bias correction is applied for CV-19 Threat betweengroup differences, the differences between the healthy group and comorbid group are marginally significant $(p=0.059)$; differences between the PTSD group and comorbid group are significant $(p=0.041)$, and differences between the chronic pain group and comorbid group are non-significant $(p=0.162)$. There was no main linear effect of authenticity, $F(1,100)=0.29, p=0.592$, partial $\eta^{2}=-0.01$, which did not support our hypothesis. Moreover, the quadratic term of authenticity was non-significant, $F(1,100)=0.61, p=0.437$, partial $\eta^{2}=-0.00$, as was the main effect of age, $F(1,100)=0.17, p=0.684$, partial $\eta^{2}=-0.01$.

We also hypothesized that the relationship between authenticity and CV-19 Threat would be dependent on group, with the healthy group showing the strongest relationship, followed by the chronic pain only and PTSD only groups, and 
TABLE 2 | Estimated marginal means and Tukey's HSD comparisons.

\begin{tabular}{lcc}
\hline Condition & CV-19 Threat Mean [95\% Cl] & CV-19 Impact Mean [95\% Cl] \\
\hline Healthy & $27.97[24.71,31.23]^{\#}$ & $30.69[26.37,35.00]$ \\
Chronic Pain & $28.97[25.23,32.71]$ & $30.41[25.46,35.35]$ \\
PTSD & $26.98[23.10,30.86]^{\star}$ & $32.34[27.21,37.47]$ \\
Comorbid & $34.95[30.53,39.37]^{\# *}$ & $43.83[37.99,49.67]^{\star \star}$
\end{tabular}

*Indicates significant between-group differences of CV-19 Threat at $p<0.05$; \# indicates marginally significant between-group differences of CV-19 Threat at $p<0.10$; ${ }^{*}$ Comorbid condition endorsed significantly greater CV-19 Impact than all other groups at $p<0.05$; there were no other between-group differences for CV-19 Impact.

then the comorbid group. Although we did not find this specific pattern, we did find that the authenticity and CV-19 Threat relationship was dependent on group. A marginally significant group by authenticity interaction emerged, $F(3,100)=2.50$, $p=0.064$, partial $\eta^{2}=0.04$. Regression analyses showed that the relationship between authenticity and CV-19 Threat significantly differed between the healthy group and chronic pain only group, $b=-0.50$, S.E. $=0.21, p=0.019,95 \%$ CI: $-0.91,-0.09, \beta=-0.30$. A simple slopes analysis showed that for those in the chronic pain only group, as authenticity increased, CV-19 Threat decreased, $b=-0.57$, S.E. $=0.16, p=0.001$. This same relationship was not seen in the other groups ( $p s>0.05)$. The overall model was significant, $F(9,100)=2.88, p=0.005$, with an adjusted $R^{2}=0.13$. See Table 3 for regression estimates.

\section{CV-19 Impact}

With CV-19 Impact as the outcome of interest, a significant main effect of group emerged, $F(3,100)=5.37, p=0.002$, partial $\eta^{2}=0.11$. Regression analyses showed that individuals in the comorbid group, compared to individuals in the healthy group, endorsed more CV-19 Impact $(b=13.14$, S.E. $=3.68, p=0.001$, 95\% CI: $5.92,20.36, \beta=0.40)$. The chronic pain only group $(b=-0.28$, S.E. $=3.45, p=0.935,95 \%$ CI: $-7.05,6.49, \beta=-$ $0.01)$ and PTSD only group $(b=1.65$, S.E. $=3.35, p=0.623$, 95\% CI: $-4.92,8.22, \beta=0.05)$ did not differ from the healthy group. Estimated marginal means showed the same significance pattern when using Tukey's HSD bias correction (see Table 2). Our hypothesis was not supported, as there was no main effect of linear authenticity, $F(1,100)=2.44, p=0.122$, partial $\eta^{2}=0.01$. Quadratic authenticity, $F(1,100)=1.29, p=0.260$, partial $\eta^{2}=0.00$ and age were non-significant, $F(1,100)=0.00, p=0.996$, partial $\eta^{2}=-0.01$. The group by authenticity interaction was also non-significant, $F(3,100)=0.58, p=0.626$, partial $\eta^{2}=-$ 0.01 , which also did not support our hypothesis of authenticity being differentially related to CV-19 Impact relevant to group. The overall model was significant, $F(9,100)=3.56, p=0.001$, with an adjusted $R^{2}=0.17$. See Table 4 for regression estimates.

\section{Correlation Analyses}

Correlation analyses collapsing across participant groups revealed the general relationships between variables included in the present study. Higher levels of authenticity were significantly related to less CV-19 Impact, $r(108)=-0.25, p=0.010$, and less severe PTSD symptoms, $r(108)=-0.49, p<0.001$. Higher levels of authenticity were marginally significantly related to lower levels of CV-19 Threat, $r(108)=-0.18, p=0.061$, and pain-related disability, $r(108)=-0.18, p=0.058$, and older age, $r(108)=0.17, p=0.079$. Higher levels of CV-19 Threat were significantly associated with higher levels of CV-19 Impact, $r(108)=0.49, p<0.001$, and pain severity $r(108)=0.21$, $p=0.025$, and marginally significantly related to more severe PTSD symptoms, $r(108)=0.17, p=0.081$. Being impacted by CV19 was significantly associated with more pain-related disability, $r(108)=0.21, p=0.028$, PTSD symptoms, $r(108)=0.37$, $p<0.001$, and pain severity, $r(108)=0.26, p=0.005$. See Table 5 for all correlations.

\section{DISCUSSION}

The present study highlights how individuals in a comorbid chronic pain and PTSD group endorse more CV-19 Threat and Impact compared to healthy individuals, those with chronic pain only, and those with PTSD only. These findings are comparable to prior research by Benedict et al. (2020) wherein individuals with

TABLE 3 | Regression estimates - CV-19 Threat.

\begin{tabular}{|c|c|c|c|c|c|}
\hline Predictor & Estimate & Est. 95\% Cl & Std. Error & $t$-statistic & $p$ \\
\hline Intercept & 28.65 & {$[25.01,32.29]$} & 1.86 & 15.44 & $<0.001^{*}$ \\
\hline \multicolumn{6}{|l|}{ Condition } \\
\hline Chronic Pain & 1.00 & {$[-4.12,6.12]$} & 2.61 & 0.38 & 0.703 \\
\hline PTSD & -0.99 & {$[-5.96,3.98]$} & 2.54 & -0.39 & 0.697 \\
\hline Comorbid & 6.98 & {$[1.52,12.43]$} & 2.78 & 2.51 & $0.014^{*}$ \\
\hline Authenticity (Linear) & -0.07 & {$[-0.32,0.18]$} & 0.13 & -0.54 & 0.592 \\
\hline Authenticity (Quadratic) & 0.00 & {$[-0.01,0.01]$} & 0.00 & -0.78 & 0.437 \\
\hline Age & 0.03 & {$[-0.12,0.18]$} & 0.08 & 0.41 & 0.684 \\
\hline \multicolumn{6}{|l|}{ Interactions } \\
\hline Authenticity ${ }^{\star}$ Chronic Pain & -0.50 & {$[-0.91,-0.09]$} & 0.21 & -2.39 & $0.019^{\star}$ \\
\hline AuthenticityPTSD & -0.02 & {$[-0.38,0.34]$} & 0.18 & -0.12 & 0.909 \\
\hline Authenticity ${ }^{\star}$ Comorbid & 0.13 & {$[-0.27,0.53]$} & 0.20 & 0.64 & 0.523 \\
\hline
\end{tabular}

Healthy condition modeled as reference group; * $p<0.05$. 
TABLE 4 | Regression estimates - CV-19 Impact.

\begin{tabular}{|c|c|c|c|c|c|}
\hline Predictor & Estimate & Est. 95\% Cl & Std. Error & $t$-statistic & $p$ \\
\hline Intercept & 31.97 & {$[23.55,40.40]$} & 4.30 & 7.44 & $<0.001^{*}$ \\
\hline \multicolumn{6}{|l|}{ Condition } \\
\hline Chronic Pain & -0.28 & {$[-7.05,6.49]$} & 3.45 & -0.08 & 0.935 \\
\hline PTSD & 1.65 & {$[-4.92,8.22]$} & 3.35 & 0.49 & 0.623 \\
\hline Comorbid & 13.14 & {$[5.92,20.36]$} & 3.68 & 3.57 & $0.001^{\star}$ \\
\hline Authenticity (Linear) & -0.26 & {$[-0.59,0.07]$} & 0.17 & -1.56 & 0.122 \\
\hline Authenticity (Quadratic) & -0.01 & {$[-0.02,0.00]$} & 0.01 & -1.13 & 0.260 \\
\hline Age & 0.00 & {$[-0.19,0.20]$} & 0.10 & 0.01 & 0.996 \\
\hline \multicolumn{6}{|l|}{ Interactions } \\
\hline Authenticity ${ }^{\star}$ Chronic Pain & -0.02 & {$[-0.56,0.52]$} & 0.28 & -0.06 & 0.954 \\
\hline Authenticity ${ }^{\star}$ PTSD & 0.27 & {$[-0.21,0.75]$} & 0.24 & 1.10 & 0.274 \\
\hline Authenticity ${ }^{\star}$ Comorbid & 0.03 & {$[-0.50,0.55]$} & 0.27 & 0.10 & 0.925 \\
\hline
\end{tabular}

Healthy condition modeled as reference group; * $p<0.05$.

comorbid chronic pain and PTSD endorsed more depression, pain severity, and pain-related disability compared to individuals with chronic pain only. Our findings also show how authenticity may have protected individuals in the chronic pain only group from CV-19 Threat compared to those in the healthy group.

We hypothesized that authenticity would attenuate the CV19 effects dependent on group. Regarding CV-19 Threat, the main effect of authenticity was non-significant, suggesting that authenticity was not a resilience factor overall for CV-19 Threat. However, results suggested that authenticity as a resilience factor of CV-19 Threat was unique to individuals with chronic pain in the absence of PTSD. The reason for null associations between authenticity and CV-19 Threat in the healthy group, PTSD only group, and comorbid group is unclear. CV-19 Threat represents anxiety and worry related to the CV-19 pandemic (Conway et al., 2020). Thus, the construct is cognitively and emotionally driven. Healthy individuals have many skills at their disposal un-disrupted by pain or PTSD (e.g., positivism and cognitive re-appraisal). Importantly, these unencumbered skills combined with lower allostatic load may make authentic living less of a factor in helping manage novel stressors for these individuals. Comparatively, individuals with chronic pain have potentially experienced inauthenticity for years due to the effects of their pain. As a result, individuals with chronic pain may be more likely to recognize authenticity as an important component of psychological health, monitor their feelings of authenticity

TABLE 5 | Correlations between variables of interest.

\begin{tabular}{lccccccr}
\hline Variable & $\mathbf{1}$ & $\mathbf{2}$ & $\mathbf{3}$ & $\mathbf{4}$ & $\mathbf{5}$ & $\mathbf{6}$ & $\mathbf{7}$ \\
\hline 1. CV-19 Threat & - & & & & & & \\
2. CV-19 Impact & $0.49^{\star}$ & - & & & & & \\
3. Authenticity & $-0.18^{\#}$ & $-0.25^{\star}$ & - & & & & \\
4. Pain-Related Disability & 0.14 & $0.21^{\star}$ & $-0.18^{\#}$ & - & & & \\
5. PTSD Symptoms & $0.17^{\#}$ & $0.37^{\star}$ & $-0.49^{*}$ & $0.32^{\star}$ & - & \\
6. Pain Severity & $0.21^{\star}$ & $0.26^{*}$ & -0.09 & $0.75^{\star}$ & $0.31^{*}$ & - & \\
7. Age & 0.01 & -0.06 & $0.17^{\#}$ & $0.35^{\star}$ & $-0.16^{\#}$ & $0.25^{\star}$ & - \\
\hline
\end{tabular}

${ }^{*} p<0.05 ;{ }^{*} p \leq 0.10$. (or lack thereof), and alter their cognitions, behaviors, and/or emotions when they do not feel authentic. While chronic pain may indeed impact how individuals perceive the world, PTSD is defined by alterations in mood and cognitions (American Psychiatric Association [APA], 2013). These alterations may prevent the use of effective self-affirming coping strategies (Vail et al., 2019, 2020) and thus, preventing individuals from harnessing the positive effects of living authentically. Moreover, authenticity is predicated on the feeling of being authentic and the ability to engage in behaviors that project those feelings (Wood et al., 2008; Lenton et al., 2013). The psychological need for feeling authentic is rooted in the idea that each individual has a primary "true self" that determines who they are, a concept known as psychological essentialism (Rivera et al., 2019). Due to the effects of pain, individuals with chronic pain may have been already altering their engagement with themselves and others prior to the pandemic, and managing what it means to be their "true self." It is possible that aspects of PTSD, such as emotional numbing, avoidance of emotions, or distrust of self and others (Ehlers and Clark, 2000), prevent individuals from benefiting from feeling authentic and/or developing a new authentic "true self." Indeed, authenticity is partially dependent on the feeling of authenticity (Wood et al., 2008; Lenton et al., 2013). If individuals with PTSD avoid certain stimuli, do not fully engage emotionally and cognitively with themselves or others, and struggle to trust themselves or others, it is more likely that they will not engage authentically and have a sense of their "true self." The present work suggests that, although individuals with chronic pain may also struggle to recognize their emotions (Aaron et al., 2019), the addition of PTSD adds a layer that may prevent one's authentic self from buffering CV-19 Threat.

Qualitative research and theoretical considerations highlight how individuals with chronic pain are forced to alter how they engage with the world, potentially causing identity-related distress (Smith and Osborn, 2007; Morley, 2008; Reed et al., 2021). Chronic pain compels individuals to find new ways of engaging authentically. If this is done effectively, then differences in authenticity between those with and without chronic pain may not be present. Also, this may account for the significant 
covariation between authenticity and CV-19 Threat in the chronic pain only group.

CV-19 Impact is conceptualized as being burdened financially or psychologically by the CV-19 pandemic (Conway et al., 2020). At first glance, authenticity did not appear to be associated with CV-19 Impact in any group. However, the standardized coefficients for all groups regarding the relationship between authenticity and CV-19 Impact ranged from -0.30 to -0.25 , except for the PTSD only group, suggesting power may be an issue (data not presented). That this relationship was only .01 in the PTSD only group supports the idea that something unique to PTSD provides a barrier to using authenticity as a resilience factor. However, this does not account for the fact the comorbid group (which includes PTSD) had a standardized beta weight of -0.25 .

Social distancing measures and protective face gear (e.g., masks) alter how to engage with others and society. For individuals with chronic pain, who are prone to higher levels of anxiety and depression (Gómez Penedo et al., 2020), there may be added anxieties around how to engage in a novel environment that the CV-19 pandemic imposes. However, it may also provide a means to re-evaluate current circumstances and engage in life in a new and more adaptive manner (Bland, 2020).

\section{Clinical Implications}

The present research also suggests that focusing on authenticity among chronic pain patients may be clinically beneficial. Non-pharmacological behavioral treatments often target pain symptoms through the acceptance of pain, altering pain-specific thoughts, and creating narratives around their pain that are ego-syntonic (Williams et al., 2012; Carr et al., 2017; Hughes et al., 2017; Chow and Fok, 2020). A common thread in these treatments is the pursuit of self-transcendence and a search for meaning. The search for transcendence is the search for meaning in something outside of the self (e.g., volunteering and helping others, spirituality, and/or religion; Wong, 2020). Pandemics and other adverse events may limit these kinds of opportunities, making the search for transcendence more relevant (Wong, 2020). Congruent with this perspective, there has been a recent push to incorporate spiritual and existential themes into the treatment of chronic pain (Dezutter et al., 2016). Indeed, patients with chronic pain note that existential satisfaction is related to pain severity and disability (Dezutter et al., 2016). A recent clinical trial showed that pain-related disability improved when an existential component was added to cognitive-behavioral therapy, compared to cognitive-behavioral therapy alone (Gebler and Maercker, 2014).

Existential-humanistic perspectives incorporate both the joyful (e.g., accomplishments, love, and awe) and anxietyprovoking realities of being human (e.g., death, sickness, and suffering) into their conceptualization (Schneider, 2008; Schneider and Längle, 2012; Wong, 2021). For instance, the novel existential positive psychology perspective posits that well-being is the result of self-transcendence, and selftranscendence means simultaneously considering one's suffering and accomplishments (Wong, 2021). Similarly, individuals with chronic pain often experience shame as a result of their physical pain (Smith and Osborn, 2007). From an existential positive psychology perspective, well-being (and "wholeness") rely on acknowledging this physical pain and corresponding suffering while taking responsibility for finding new ways to engage meaningfully in life, connect with others, and maintain a spiritual and/or philosophical component of life (Wong, 2018, 2021). Therapy, then, can focus on finding ways to engage authentically while targeting these themes.

Other approaches offer different perspectives but remain faithful to not avoiding the realities of human suffering and physical pain. Research suggests acceptance and commitment therapy (ACT) and mindfulness approaches have shown to be effective at treating chronic pain outcomes (Veehof et al., 2011). ACT is a values-based approach that engages patients in determining what is important to them and then focuses on acting on these values (Hayes et al., 2012). In this way, ACT encourages individuals to engage authentically with the world and others. Mindfulness approaches are presentfocused and teach individuals to "let go" of thoughts and emotions as being truly representative of themselves. In this way, mindfulness approaches de-emphasize rigid thought patterns and allow individuals to get more in tune with their authentic self.

\section{Limitations and Future Directions}

The present study has limitations and future directions. As a cross-sectional design, causality cannot be established, and longitudinal designs attempting to parse out causal effects are indicated. With only 110 individuals completing Time 2 , the null results may also be due to insufficient power (as suggested above). Although we intended to recruit individuals who were diagnosed with chronic pain and PTSD, standardized clinical interviews were not completed. Thus, it is possible that individuals would not qualify for these diagnoses through clinical interviews. The study sample lacked diversity regarding race, sexual orientation, and education. Previous research shows that people of color and lower social-economic status are more severely affected by the pandemic (Czeisler et al., 2020). The present study's results should be interpreted in light of these generalizability restrictions. There is a plethora of evidence showing how the current medical systems in place disproportionately negatively affect certain marginalized populations (Liburd et al., 2020). The present work generally fails to include these populations, and more research is indicated to better understand the impact of the pandemic within these populations. Using a $21 \%$ pain disability cutoff score for the chronic pain only and comorbid group, combined with the average pain severity scores of 3.65 (chronic pain only) and 3.59 (comorbid group) point to a sample that is not as severe as other chronic pain populations. Similarly, the average PTSD scores for the PTSD only group were 30.09, and the average score for the comorbid chronic pain and PTSD only group was 38.23. These scores indicate the sample may not be as severe as other PTSD populations. Future research should include participants who are more severe, as results in these populations may vary from present results. Finally, correlations are presented with the caveat that the study design was such that specific groups of individuals were targeted for recruitment. Specifically, each participant group reflects a limited range of pain severity and 
PTSD symptoms. Therefore, interpreting correlations involving pain severity, pain-related disability, and PTSD symptoms in individuals who do not have chronic pain and/or PTSD is of limited utility.

\section{CONCLUSION}

The present study examined authenticity as a resilience factor during the CV-19 pandemic. Participants were allocated to four separate groups based on their level of pain disability and PTSD symptom severity: healthy, chronic pain only, PTSD only, and comorbid chronic pain and PTSD. The comorbid group endorsed higher levels of CV-19 Threat and Impact compared to all other groups. There were no other between-group differences in CV-19 Threat and Impact. Importantly, greater authenticity was associated with less CV-19 Threat in the chronic pain only group, and not in any other group. As non-pharmacological chronic pain therapies focused on values, identity, acceptance, and internal narratives gain more popularity, incorporating elements designed to boost authenticity may play an important role. Results are interpreted within the limits of generalizability, as the present sample included a majority of White and educated participants.

\section{REFERENCES}

Aaron, R. V., Fisher, E. A., de la Vega, R., Lumley, M. A., and Palermo, T. M. (2019). Alexithymia in individuals with chronic pain and its relation to pain intensity, physical interference, depression, and anxiety. Pain 160, 994-1006. doi: 10.1097/j.pain.0000000000001487

American Psychiatric Association [APA] (2013). Diagnostic and Statistical Manual of Mental Disorders, 5th Edn. Washington, D.C: American Psychiatric Association.

Bell, V., Robinson, B., Katona, C., Fett, A.-K., and Shergill, S. (2019). When trust is lost: the impact of interpersonal trauma on social interactions. Psychol. Med. 49, 1041-1046. doi: 10.1017/S0033291718001800

Benedict, T. M., Keenan, P. G., Nitz, A. J., and Moeller-Bertram, T. (2020). Post-traumatic stress disorder symptoms contribute to worse pain and health outcomes in veterans with PTSD compared to those without: a systematic review with meta-analysis. Mil. Med. 185, e1481-e1491. doi: 10.1093/milmed/ usaa052

Blaettler, L. T., Stewart, J. A., Gubler, D. A., Egloff, N., von Känel, R., and Grosse Holtforth, M. (2019). Alexithymia moderates effects of psychotherapeutic treatment expectations on depression outcome in interdisciplinary chronic pain treatment. J. Psychosom. Res. 122, 69-72. doi: 10.1016/j.jpsychores.2019.04.010

Bland, A. M. (2020). Existential givens in the COVID-19 crisis. J. Hum. Psychol. 60, 710-724. doi: 10.1177/0022167820940186

Blevins, C. A., Weathers, F. W., Davis, M. T., Witte, T. K., and Domino, J. L. (2015). The posttraumatic stress disorder checklist for DSM-5 (PCL-5): development and initial psychometric evaluation. J. Trauma. Stress 28, 489-498. doi: 10.1002/ jts. 22059

Boyraz, G., Waits, J. B., and Felix, V. A. (2014). Authenticity, life satisfaction, and distress: a longitudinal analysis. J. Couns. Psychol. 61, 498-505. doi: 10.1037/ cou0000031

Bryan, J. L., Baker, Z. G., and Tou, R. Y. (2017). Prevent the blue, be true to you: authenticity buffers the negative impact of loneliness on alcohol-related problems, physical symptoms, and depressive and anxiety symptoms. J. Health Psychol. 22, 605-616. doi: 10.1177/1359105315609090

Carr, E. C. J., McCaffrey, G., and Ortiz, M. M. (2017). The suffering of chronic pain patients on a wait list: are they amenable to narrative therapy? Can. J. Pain 1, 14-21. doi: 10.1080/24740527.2017.1316173

\section{DATA AVAILABILITY STATEMENT}

The original contributions presented in the study are publicly available. This data can be found here: https://osf.io/zub97/.

\section{ETHICS STATEMENT}

The studies involving human participants were reviewed and approved by the University of Texas Health Science Center at San Antonio. Written informed consent for participation was not required for this study in accordance with the national legislation and the institutional requirements.

\section{AUTHOR CONTRIBUTIONS}

DR contributed to research design, data collection, data analysis, and manuscript preparation. EL, BC, KV, PN, PH, and MG contributed to research design and manuscript preparation. DM contributed to data collection and research design. All authors contributed to the article and approved the submitted version.

Chow, E. O. W., and Fok, D. Y. H. (2020). Recipe of life: a relational narrative approach in therapy with persons living with chronic pain. Res. Soc. Work Pract. 30, 320-329. doi: 10.1177/1049731519870867

Cleeland, C. S. (1991). Brief Pain Inventory (Short Form). Pain Research Group. Available online at: https://www.mdanderson.org/documents/Departmentsand-Divisions/Symptom-Research/BPI_UserGuide.pdf (accessed April 13, 2021).

Conway, L. G., Woodard, S. R., and Zubrod, A. (2020). Social Psychological Measurements of COVID-19: Coronavirus Perceived Threat, Government Response, Impacts, and Experiences Questionnaires. PsyArXiv [Preprint] doi: 10.31234/osf.io/z2x9a.

Czeisler, M. É, Lane, R. I., Petrosky, E., Wiley, J. F., Christensen, A., Njai, R., et al. (2020). Mental health, substance use, and suicidal ideation during the COVID19 pandemic - United States, June 24-30, 2020. MMWR. Morb. Mortal. Wkly Rep. 69, 1049-1057. doi: 10.15585/mmwr.mm6932a1

Davis, W. E., and Hicks, J. A. (2013). Maintaining hope at the 11th hour: authenticity buffers the effect of limited time perspective on hope. Pers. Soc. Psychol. Bull. 39, 1634-1646. doi: 10.1177/0146167213500150

De Heer, E. W., Gerrits, M. M. J. G., Beekman, A. T. F., Dekker, J., Van Marwijk, H. W. J., De Waal, M. W. M., et al. (2014). The association of depression and anxiety with pain: a study from NESDA. PLoS One 9:e106907. doi: 10.1371/ journal.pone.0106907

Dezutter, J., Offenbaecher, M., Vallejo, M. A., Vanhooren, S., Thauvoye, E., and Toussaint, L. (2016). Chronic pain care: the importance of a biopsychosocialexistential approach. Int. J. Psychiatry Med. 51, 563-575. doi: 10.1177/ 0091217417696738

Ehlers, A., and Clark, D. M. (2000). A cognitive model of posttraumatic stress disorder. Behav. Res. Ther. 38, 319-345. doi: 10.1016/S0005-7967(99)00123-0

Fairbank, J. C., Couper, J., Davies, J. B., and O’Brien, J. P. (1980). The Oswestry low back pain disability questionnaire. Physiotherapy 66, 217-273.

Feinberg, R. K., Hu, J., Weaver, M. A., Fillingim, R. B., Swor, R. A., Peak, D. A., et al. (2017). Stress-related psychological symptoms contribute to axial pain persistence after motor vehicle collision. Pain 158, 682-690. doi: 10.1097/j.pain. 0000000000000818

Fritz, J. M., and Irrgang, J. J. (2001). A comparison of a modified Oswestry low back pain disability questionnaire and the Quebec back pain disability scale. Phys. Therapy 81, 776-788. doi: 10.1093/ptj/81.2.776 
Gebler, F. A., and Maercker, A. (2014). Effects of including an existential perspective in a cognitive-behavioral group program for chronic pain: a clinical trial with 6 months follow-up. Humanist. Psychol. 42, 155-171. doi: 10.1080/ 08873267.2013.865188

Gómez Penedo, J. M., Rubel, J. A., Blättler, L., Schmidt, S. J., Stewart, J., Egloff, N., et al. (2020). The complex interplay of pain, depression, and anxiety symptoms in patients with chronic pain. Clin. J. Pain 36, 249-259. doi: 10.1097/AJP. 0000000000000797

Grijak, D. (2017). Authenticity as a predictor of mental health. Klinička Psihologija 10, 23-34. doi: 10.21465/2017-KP-1-2-0002

Grühn, D., Sharifian, N., and Chu, Q. (2016). The limits of a limited future time perspective in explaining age differences in emotional functioning. Psychol. Aging 31, 583-593. doi: 10.1037/pag0000060

Guo, J., Feng, X. L., Wang, X. H., and van IJzendoorn, M. H. (2020). Coping with COVID-19: exposure to covid-19 and negative impact on livelihood predict elevated mental health problems in Chinese adults. Int. J. Environ. Res. Public Health 17:3857. doi: 10.3390/ijerph17113857

Hayes, S. C., Strosahk, K. D., and Wilson, K. G. (2012). Acceptance and Commitment Therapy: The Process and Practice of Mindful Change, 2nd Edn. New York, NY: Guilford Press.

Hughes, L. S., Clark, J., Colclough, J. A., Dale, E., and McMillan, D. (2017). Acceptance and commitment therapy (ACT) for chronic pain. Clin. J. Pain 33, 552-568. doi: 10.1097/AJP.0000000000000425

Jones, K. R., Vojir, C. P., Hutt, E., and Fink, R. (2007). Determining mild, moderate, and severe pain equivalency across pain-intensity tools in nursing home residents. J. Rehabil. Res. Dev. 44:305. doi: 10.1682/JRRD.2006.05.0051

Kalisch, R., Baker, D. G., Basten, U., Boks, M. P., Bonanno, G. A., Brummelman, E., et al. (2017). The resilience framework as a strategy to combat stress-related disorders. Nat. Hum. Behav. 1, 784-790. doi: 10.1038/s41562-017-0200-8

Kalisch, R., Cramer, A. O. J., Binder, H., Fritz, J., Leertouwer, I., Lunansky, G., et al. (2019). Deconstructing and reconstructing resilience: a dynamic network approach. Perspect. Psychol. Sci. 14, 765-777. doi: 10.1177/1745691619855 637

Lenton, A. P., Bruder, M., Slabu, L., and Sedikides, C. (2013). How does "being real" feel? The experience of state authenticity. J. Pers. 81, 276-289. doi: 10.1111/j. 1467-6494.2012.00805.x

Liburd, L. C., Hall, J. E., Mpofu, J. J., Williams, S. M., Bouye, K., and Penman-Aguilar, A. (2020). Addressing health equity in public health practice: frameworks, promising strategies, and measurement considerations. Annu. Rev. Public Health 41, 417-432. doi: 10.1146/annurev-publhealth-040119-094119

Litman, L., Robinson, J., and Abberbock, T. (2017). TurkPrime.com: a versatile crowdsourcing data acquisition platform for the behavioral sciences. Behav. Res. Methods 49, 433-442. doi: 10.3758/s13428-016-0727-z

Liu, C. H., Zhang, E., Wong, G. T. F., Hyun, S., and Hahm, H. (2020). Factors associated with depression, anxiety, and PTSD symptomatology during the COVID-19 pandemic: clinical implications for U.S. young adult mental health. Psychiatry Res. 290:113172. doi: 10.1016/j.psychres.2020.113172

Maffly-Kipp, J., Flanagan, P., Kim, J., Schlegel, R. J., Vess, M., and Hicks, J. A. (2020). The role of perceived authenticity in psychological recovery from collective trauma. J. Soc. Clin. Psychol. 39, 419-448. doi: 10.1521/jscp.2020.39.5. 419

McEwen, B. S. (1998). Stress, adaptation, and disease: allostasis and allostatic load. Ann. NY Acad. Sci. 840, 33-44. doi: 10.1111/j.1749-6632.1998.tb09546.x

McGeary, C., Nabity, P., Reed, D., Cobos, B., Eapen, B., Pugh, M. J., et al. (2020). A test of the fear avoidance model to predict chronic pain outcomes in a polytrauma sample. NeuroRehabilitation 47, 35-43. doi: 10.3233/NRE-203084

McWilliams, L. A., Cox, B. J., and Enns, M. W. (2003). Mood and anxiety disorders associated with chronic pain: an examination in a nationally representative sample. Pain 106, 127-133. doi: 10.1016/S0304-3959(03)00301-4

Merskey, H. and Bogduk, N. (1994). Classification of Chronic Pain, 2nd Edn, IASP Task Force on Taxonomy. Seattle, WA: IASP Press.

Michigan State University (2020). Oswestry Low Back Pain Disability Questionnaire. Michigan State University: East Lansing, MI.

Mitchell, L. L., Frazier, P. A., and Sayer, N. A. (2020). Identity disruption and its association with mental health among veterans with reintegration difficulty. Dev. Psychol. 56, 2152-2166. doi: 10.1037/dev0001106

Morley, S. (2008). Psychology of pain. Br. J. Anaesthesia 101, 25-31. doi: 10.1093/ bja/aen123
Packham, T. L., Wainio, K., and Wong, M. K. (2020). Persons with complex regional pain syndrome renegotiate social roles and intimacy: a qualitative study. Pain Med. 21, 239-246. doi: 10.1093/pm/pnz173

Pfefferbaum, B., and North, C. S. (2020). Mental health and the Covid-19 pandemic. N. Engl. J. Med. 383, 510-512.

Pyszczynski, T., Lockett, M., Greenberg, J., and Solomon, S. (2020). Terror management theory and the COVID-19 pandemic. J. Hum. Psychol. 61:002216782095948. doi: 10.1177/0022167820959488

Reed, D. E. (2020). Future time perspective as an operationalization of existential concerns related to time. J. Hum. Psychol. 1-14. doi: 10.1177/ 0022167820945067

Reed, D. E. II, Cobos, B., Nabity, P. S., Doolin, J., and McGeary, D. D. (2021). "Comorbid chronic pain and posttraumatic stress disorder: Current knowledge, treatments, and future directions," in Pain Care Esential and Innovations, eds S. Pangarkar, Q. G. Pham, and B. C. Eapen (Amsterdam: Elsevier), 211-227.

Rivera, G. N., Christy, A. G., Kim, J., Vess, M., Hicks, J. A., and Schlegel, R. J. (2019). Understanding the relationship between perceived authenticity and well-being. Rev. Gen. Psychol. 23, 113-126. doi: 10.1037/gpr0000161

Rodriguez, L. M., Litt, D. M., and Stewart, S. H. (2020). Drinking to cope with the pandemic: the unique associations of COVID-19-related perceived threat and psychological distress to drinking behaviors in American men and women. Addict. Behav. 110:106532. doi: 10.1016/j.addbeh.2020.106532

Ryder, A. L., Azcarate, P. M., and Cohen, B. E. (2018). PTSD and physical health. Curr. Psychiatry Rep. 20:116. doi: 10.1007/s11920-018-0977-9

Schneider, K. J. (ed.) (2008). "From segregation to integration," in ExistentialIntegrative Psychotherapy: Guideposts to the Core of Practice, (New York, NY: Routledge), 15-22.

Schneider, K. J., and Längle, A. (2012). The renewal of humanism in psychotherapy: a roundtable discussion. Psychotherapy 49, 427-429. doi: 10.1037/a0027111

Serlin, R. C., Mendoza, T. R., Nakamura, Y., Edwards, K. R., and Cleeland, C. S. (1995). When is cancer pain mild, moderate or severe? Grading pain severity by its interference with function. Pain 61, 277-284. doi: 10.1016/0304-3959(94) 00178-H

Sharp, T. J., and Harvey, A. G. (2001). Chronic pain and posttraumatic stress disorder: mutual maintenance? Clin. Psychol. Rev. 21, 857-877. doi: 10.1016/ S0272-7358(00)00071-4

Sheldon, K. M., Ryan, R. M., Rawsthorne, L. J., and Ilardi, B. C. (1997). Trait self and true self: cross-role variation in the big-five personality traits and its relations with psychological authenticity and subjective well-being. J. Pers. Soc. Psychol. 73, 1380-1393. doi: 10.1037/0022-3514.73.6.1380

Smith, J. A., and Osborn, M. (2007). Pain as an assault on the self: an interpretative phenomenological analysis of the psychological impact of chronic benign low back pain. Psychol. Health 22, 517-534. doi: 10.1080/14768320600941756

Sterling, P., and Eyer, J. (1988). "Allostasis: a new paradigm to explain arousal pathology," in Handbook of Life Stress, Cognition and Health, eds S. Fisher and J. Reason (Hoboken, NJ: ). John Wiley \& Sons), 629-649.

Treede, R.-D., Rief, W., Barke, A., Aziz, Q., Bennett, M. I., Benoliel, R., et al. (2015). A classification of chronic pain for ICD-11. PAIN 156, 1003-1007. doi: $10.1097 /$ j.pain. 0000000000000160

U.S. Department of Veterans Affairs (2020). PTSD Checklist for DSM-5 (PCL-5). Washington, D.C: U.S. Department of Veterans Affairs.

Vail, K. E., Goncy, E. A., and Edmondson, D. (2019). Anxiety buffer disruption: worldview threat, death thought posttraumatic stress symptom samples. Psychol. Trauma. 11, 647-655. doi: 10.1037/tra000441

Vail, K. E., Juhl, J., Arndt, J., Vess, M., Routledge, C., and Rutjens, B. T. (2012). When death is good for life. Pers. Soc. Psychol. Rev. 16, 303-329. doi: 10.1177/ 1088868312440046

Vail, K. E., Reed, D. E., Goncy, E. A., Cornelius, T., and Edmondson, D. (2020). Anxiety buffer disruption: self-evaluation, death anxiety, and stressor appraisals among low and high posttraumatic stress symptom samples. J. Soc. Clin. Psychol. 39, 353-382. doi: 10.1521/jscp.2020.39.5.353

Veehof, M. M., Oskam, M.-J., Schreurs, K. M. G., and Bohlmeijer, E. T. (2011). Acceptance-based interventions for the treatment of chronic pain: a systematic review and meta-analysis. Pain 152, 533-542. doi: 10.1016/j.pain.2010.11.002

Vess, M., Leal, S. A., Hoeldtke, R. T., Schlegel, R. J., and Hicks, J. A. (2016). True self-alienation positively predicts reports of mindwandering. Conscious. Cogn. 45, 89-99. doi: 10.1016/j.concog.2016.08.018 
Weathers, F. W., Litz, B. T., Keane, T. M., Palmieri, P. A., Marx, B. P., and Schnurr, P. P. (2013). The PTSD Checklist for DSM-5 (PCL-5). National Center for PTSD, 5, 2002. Available online at: https://doi.org/doi:10.1037/t02622-000 (accessed April 13, 2021).

Williams, A., Eccleston, C., and Morley, S. (2012). Psychological therapies for the management of chronic pain (excluding headache) in adults. Cochrane Database Syst. Rev. 11:CD007407. doi: 10.1002/14651858.CD007407.pub3

Wong, P. (2018). From Shame to Wholeness: An Existential Positive Psychology Perspective. Available online at: http://www.drpaulwong.com/from-shame-towholeness-an-existential-positive-psychology-perspective/ (accessed April 13, 2021).

Wong, P. (2021). What is Existential Positive Psychology (PP 2.0)? Why is it Necessary for Mental Health During the Pandemic?. Available online at: http://www.drpaulwong.com/what-is- existential-positive-psychology-whyis-it-necessary-for-mental-health-during-the-pandemic/ (accessed April 13, 2021).

Wong, P. T. P. (2020). Existential positive psychology and integrative meaning therapy. Int. Rev. Psychiatry 32, 565-578. doi: 10.1080/09540261.2020.181 4703
Wood, A. M., Linley, P. A., Maltby, J., Baliousis, M., and Joseph, S. (2008). The authentic personality: a theoretical and empirical conceptualization and the development of the authenticity scale. J. Counsel. Psychol. 55, 385-399. doi: 10.1037/0022-0167.55.3.385

World Health Organization [WHO] (2020). World Health Organization. Available online at: https://covid19.who.int/region/amro/country/us (accessed April 13, 2021).

Conflict of Interest: The authors declare that the research was conducted in the absence of any commercial or financial relationships that could be construed as a potential conflict of interest.

Copyright () 2021 Reed, Lehinger, Cobos, Vail, Nabity, Helm, Galgali and McGeary. This is an open-access article distributed under the terms of the Creative Commons Attribution License (CC BY). The use, distribution or reproduction in other forums is permitted, provided the original author(s) and the copyright owner(s) are credited and that the original publication in this journal is cited, in accordance with accepted academic practice. No use, distribution or reproduction is permitted which does not comply with these terms. 